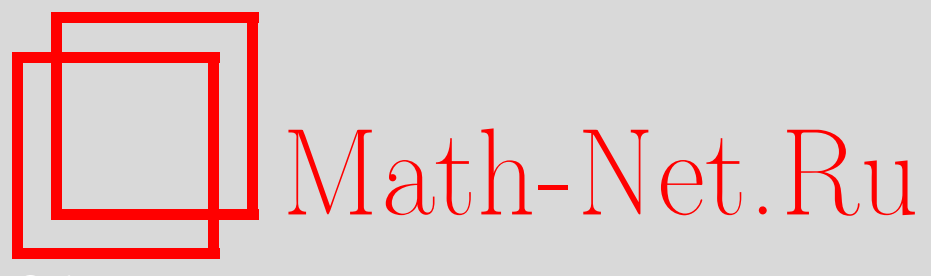

А. В. Чернов, О поточечной оценке разности решений управляемого функционально-операторного уравнения в лебеговых пространствах, Матем. заметки, 2010, том 88, выпуск 2, 288-302

DOI: https://doi.org/10.4213/mzm3898

Использование Общероссийского математического портала Math-Net.Ru подразумевает, что вы прочитали и согласны с пользовательским соглашением http: //www . mathnet.ru/rus/agreement

Параметры загрузки:

IP: 18.234 .156 .22

26 апреля 2023 г., 16:12:16

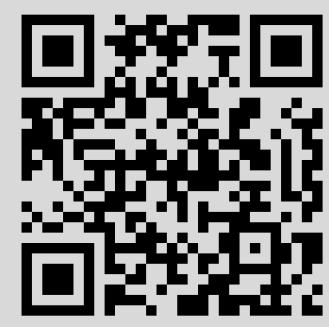


Том 88 выпуск 2 август 2010

УДК 517.988+517.977.56

\section{О поточечной оценке разности решений управляемого функционально-операторного уравнения в лебеговых пространствах}

\section{А. В. Чернов}

Для функционально-операторного уравнения в лебеговом пространстве доказывается утверждение о поточечной оценке модуля приращения глобального (на фиксированном множестве $\Pi \subset \mathbb{R}^{n}$ ) решения при варьировании входящей в это уравнение управляющей функции. В качестве вспомогательного утверждения доказывается один вариант обобщения леммы Гронуолла на случай нелинейного оператора, действующего в лебеговом пространстве. Используемый подход основан на методах теории устойчивости существования глобального решения вольтерровых операторных уравнений.

Библиография: 15 названий.

Введение. Существует довольно широкий класс управляемых начально-краевых задач, которые естественным образом (см. пример в п. 5) приводятся к функционально-операторному уравнению вида

$$
y(t)=w(t)+A[g(\cdot, y(\cdot), u(\cdot))](t), \quad t \in \Pi, \quad y(\cdot) \in L_{q}^{l}(\Pi),
$$

где $\Pi \subset \mathbb{R}^{n}$ - измеримое по Лебегу ограниченное множество, $w(\cdot) \in L_{q}^{l}(\Pi), u(\cdot) \in$ $\mathscr{D}$ - управляющая функция (управление), $\mathscr{D} \subset L_{r}^{s}(\Pi)$ - ограниченное множество, $A: L_{p}^{m}(\Pi) \rightarrow L_{q}^{l}(\Pi)$ - линейный ограниченный оператор (ЛОО), числа $n, m, l, s \in \mathbb{N}$, $1 \leqslant p<q<+\infty$ заданы, $g(\cdot, \cdot, \cdot): \Pi \times \mathbb{R}^{l} \times \mathbb{R}^{s} \rightarrow \mathbb{R}^{m}$ - заданная функция.

В данной статье формулируется и доказывается теорема, позволяющая получать поточечную (на всем множестве П) оценку по модулю приращения решения уравнения (1) при варьировании управления $u(\cdot)$.

В качестве вспомогательного утверждения доказывается один вариант нелинейного обобщения леммы Гронуолла, который состоит в следующем.

Пусть $1 \leqslant p<q<\infty, \Pi \subset \mathbb{R}^{n}, F: L_{q}(\Pi) \rightarrow L_{q}(\Pi)$ - нелинейный оператор вида $F[x]=B\left[x^{q / p}\right]$, где оператор $B: L_{p} \rightarrow L_{q}$ линейный и положительный, а функция $\varphi \in L_{q}$ неотрицательна и достаточно мала по норме. При этих условиях строится поточечная на всем множестве П оценка сверху для всякого $x \in L_{q}(\Pi)$, удовлетворяющего неравенству $x \leqslant F[x]+\varphi$. Применяемый подход ${ }^{1}$ основан на теореме

Работа выполнена при финансовой поддержке Российского фонда фундаментальных исследований (грант № 07-01-00495).

${ }^{1}$ Отметим, что он дает возможность рассмотреть и случай оператора $F$ общего вида.

(C) A. В. Чернов, 2010 
устойчивости существования глобальных решений вольтерровых операторных уравнений [1]. В связи с этим следует отметить, что различные нелинейные обобщения леммы Гронуолла и утверждения о неравенствах, позволяющих строить оценки решений операторных уравнений и, в частности, дифференциальных, интегральных и интегро-дифференциальных уравнений, доказывались и ранее. Начало этому было положено в работах С.А. Чаплыгина. Что касается операторных уравнений в пространствах функций одной переменной, эти классические результаты получили широкое развитие - укажем, в частности, работы Азбелева, Цалюка, Жуковского [2]-[4]. Имеются и более абстрактные результаты, например, [5] (там же см. библиографию).

Основное отличие нашего способа обобщения леммы Гронуолла состоит в том, что оценка $x$ строится на заранее заданном множестве П, одном и том же для любой функции $\varphi$, достаточно малой по норме, тогда как до сих пор (в сходных ситуациях) доказывались оценки, локальные по множеству изменения независимых переменных. Это обстоятельство весьма существенно, поскольку именно оно и позволяет строить равномерные поточечные оценки глобальных решений бесконечного семейства вольтерровых операторных уравнений, отвечающего некоторому ограниченному множеству управлений, при том, однако, условии, что нормы управлений отличаются достаточно мало. Соответствующий подход основан на методах теории устойчивости существования глобального решения вольтерровых операторных уравнений.

Теория достаточных условий устойчивости существования глобальных решений так называемых функциональных вольтерровых уравнений в лебеговых пространствах и функциональных вольтерровых уравнений второго рода общего вида в пространстве $L_{\infty}^{m}(\Pi)$ была построена Суминым (см., например, [6]-[8]). Им же были даны разнообразные примеры применения получаемых общих условий такой устойчивости в конкретных оптимизационных задачах. В статье [1] схема Сумина получения условий устойчивости существования глобальных решений функциональных уравнений распространена на случай вольтерровых операторных уравнений второго рода общего вида в банаховом пространстве. Конструктивность доказанных общих теорем устойчивости существования была проиллюстрирована в [1] на примере задачи Коши для гиперболического уравнения первого порядка при варьировании старшего коэффициента. При этом оценка разности решений и в работах Сумина, и в статье [1] строилась только по норме пространства. В данной статье производится, фактически, некоторая модернизация схемы доказательства теоремы устойчивости существования [1], которая и позволяет получить нелинейный аналог леммы Гронуолла.

1. Формулировка основных результатов. Далее мы везде будем предполагать, что $\Pi \subset \mathbb{R}^{n}$ - ограниченное измеримое по Лебегу множество. Обозначим $\Sigma=\Sigma(\Pi)-\sigma$-алгебра всех измеримых по Лебегу подмножеств множества $\Pi, P_{H}-$ оператор умножения на характеристическую функцию $\chi_{H}(\cdot)$ множества $H \in \Sigma$ (будем обозначать его одинаково вне зависимости от того, в каком именно пространстве $L_{p}(\Pi), p \in[1, \infty]$, он рассматривается).

Будем считать, кроме того, что числа $1 \leqslant p<q<\infty$ заданы, $L_{p}=L_{p}(\Pi), L_{q}=$ $L_{q}(\Pi)$,

$$
L_{q}^{+}=L_{q}^{+}(\Pi)=\left\{x(\cdot) \in L_{q}(\Pi): x(t) \geqslant 0 \text { для п.в. } t \in \Pi\right\} .
$$


В случае $x \in L_{p}$ норму $\|x\|_{L_{p}}$, если иное не оговорено, будем обозначать просто $\|x\|$. Пусть теперь $F: L_{p}(\Pi) \rightarrow L_{q}(\Pi)$ - некоторый оператор. Всякое множество $H \in \Sigma$ такое, что $P_{H} F P_{H}=P_{H} F$, будем называть вольтерровским множеством оператора $F$. Совокупность всех вольтерровских множеств оператора $F$ будем обозначать $\mathscr{B}(F)$.

Всякую конечную систему множеств $\left\{H_{0}, H_{1}, \ldots, H_{k}\right\} \subset \mathscr{B}(F)$, обладающую свойством

$$
\varnothing=H_{0} \subset H_{1} \subset \cdots \subset H_{k}=\Pi,
$$

назовем вольтерровской цепочкой ЛОО $F: L_{p} \rightarrow L_{q}$ и, соответственно, вольтерровской $\delta$-цепочкой этого оператора, если, кроме того,

$$
\left\|P_{h} F P_{h}\right\|<\delta \quad \text { для } \quad h=H_{i} \backslash H_{i-1}, \quad i=1, \ldots, k .
$$

Так, например, если $\Pi=\left[0, b_{1}\right] \times\left[0, b_{2}\right] \subset \mathbb{R}^{2}$, а оператор $F$ определяется формулой ${ }^{2}$

$$
F[z](t)=\int_{0}^{t_{1}} d \xi_{1} \int_{0}^{t^{2}} z(\xi) d \xi_{2}, \quad t=\left\{t_{1}, t_{2}\right\} \in \Pi, \quad z \in L_{p}(\Pi),
$$

то в качестве вольтерровской $\delta$-цепочки годится, например, система

$$
\left\{H(\tau[i]): 0=\tau[0]<\cdots<\tau[k]=b_{1}+b_{2}\right\}
$$

множеств вида

$$
H(\tau)=\left\{t=\left\{t_{1}, t_{2}\right\} \in \Pi: t_{1}+t_{2} \leqslant \tau\right\},
$$

при условии, что мелкость разбиения $\lambda=\max _{i=1, \ldots, k}|\tau[i]-\tau[i-1]|$ достаточно мала.

Обратимся к уравнению (1). Далее будем предполагать, что функция $g(\cdot, \cdot, \cdot)$ удовлетворяет следующим условиям типа Каратеодори.

Условие К). Выполнены условия

а) функция $g(t, x, w)$ непрерьвно дифференцируема по $x \in \mathbb{R}^{l}$ для n.в. $t \in \Pi u$ всех $w \in \mathbb{R}^{s}$ и вместе со своей производной $g_{x}^{\prime}(t, x, w): \Pi \times \mathbb{R}^{l} \times \mathbb{R}^{s} \rightarrow \mathbb{R}^{m \times l}$ измерима по $t \in \Pi$ и непрерьвна по $\{x, w\} \in \mathbb{R}^{l} \times \mathbb{R}^{s}$ для n.в. $t \in \Pi$;

б) дляккаждого $u(\cdot) \in L_{r}^{s}(\Pi)$ формула $\mathbf{G}_{u}[y]=g(\cdot y(\cdot), u(\cdot))$ определяет операmop $\mathbf{G}_{u}: L_{q}^{l}(\Pi) \rightarrow L_{p}^{m}(\Pi)$;

в) для каждого $u \in L_{r}^{s}(\Pi)$ формула $\mathscr{G}_{u}[y]=g_{x}^{\prime}(\cdot, y(\cdot), u(\cdot))$ определяет операmop $\mathscr{G}_{u}: L_{q}^{l}(\Pi) \rightarrow L_{\sigma}^{m \times l}(\Pi), 1 / q+1 / \sigma=1 / p$.

Относительно ЛОО $A$ будем предполагать, что выполнено 3

УСловиЕ А). Выполнены следующие условия:

а) $A$ обладает положительной мажорантой $B: L_{p}(\Pi) \rightarrow L_{q}(\Pi)$;

б) ЛОО В имеет для всех $\delta>0$ вольтерровскую $\delta$-цепочку $\mathscr{T}_{\delta}$.

Пусть $U$ - некоторое ограниченное множество в линейном нормированном пространстве (ЛНП), $E$ - другое ЛНП, $\gamma>0$. Тогда нелинейный оператор $F: U \rightarrow E$ условимся называть $\gamma$-ограниченным на $U$, если $\|F[x]\| \leqslant \gamma\|x\|$ для всех $x \in U$.

\footnotetext{
2 Это так называемый разрешающий оператор задачи Гурса-Дарбу.

${ }^{3}$ По поводу условий, гарантирующих выполнение условия б), см. [9]. Впрочем, его довольно часто удается проверить и непосредственно, см. пример выше и в п. 5.
} 
Tеорема 1.1. Пусть $V(\cdot) \in L_{r}(\Pi), v(\cdot) \in L_{r}^{s}(\Pi)$ - некоторые фиксированные функиии, причем для $u=v$ существует решение $y=y_{v}$ уравнения (1);

$$
\mathscr{V}=\left\{u(\cdot) \in L_{r}^{s}(\Pi):|u(t)| \leqslant V(t) \text { для n.в. } t \in \Pi\right\} .
$$

Тогда найдутся числа $\varepsilon>0, \gamma>0$ и $\gamma$-ограниченный оператор $S^{-1}: U_{\varepsilon}(0) \rightarrow L_{q}^{+}(\Pi)$, определенный на шаре $U_{\varepsilon}(0) \subset L_{q}^{+}(\Pi)$, такие, что справедливо следующее утверждение. Для любого решения $y=y_{u}$ уравнения (1), отвечающего некоторому $u \in \mathscr{V}$ такому, что $\left\|B\left[\left|\Delta_{u} g\left(y_{v}\right)\right|\right]\right\|<\varepsilon$, где

$$
\Delta_{u} g\left(y_{v}\right)=g\left(\cdot, y_{v}(\cdot), u(\cdot)\right)-g\left(\cdot, y_{v}(\cdot), v(\cdot)\right),
$$

имеет место поточечная оценка

$$
|\Delta y(t)|=\left|y_{u}(t)-y_{v}(t)\right| \leqslant S^{-1} B\left[\left|\Delta_{u} g\left(y_{v}\right)\right|\right] \quad \text { для п.в. } t \in \Pi .
$$

Tеорема 1.2. Пусть $V(\cdot) \in L_{r}(\Pi), v(\cdot) \in L_{r}^{s}(\Pi)$ - некоторые фиксированные функиии, причем для $u=v$ существует решение $y=y_{v}$ уравнения (1);

$$
\mathscr{V}=\left\{u(\cdot) \in L_{r}^{s}(\Pi):|u(t)| \leqslant V(t) \text { для n.в. } t \in \Pi\right\} .
$$

Пусть, кроме того, для всех достаточно малых $\varepsilon>0$ определено некоторое семейство $\mathscr{V}_{\varepsilon}$ функций из $\mathscr{V}$ такое, что

$$
z_{\varepsilon}(t) \equiv \sup _{u \in \mathscr{V}_{\varepsilon}} B\left[\left|\Delta_{u} g\left(y_{v}\right)\right|\right] \rightarrow 0 \quad \text { npu } \quad \varepsilon \rightarrow+0 \quad \text { для n.в. } t \in \Pi .
$$

Тогда для каждого достаточно малого числа $\varepsilon>0$ найдется функиия $Z_{\varepsilon}(\cdot) \in$ $L_{q}^{+}(\Pi)$ такая, что для любого решения уравнения (1) $y=y_{u}$, отвечающего некоторому $и \in \mathscr{V}_{\varepsilon}$, имеет место поточечная оценка

$$
|\Delta y(t)|=\left|y_{u}(t)-y_{v}(t)\right| \leqslant Z_{\varepsilon}(t) \quad \text { для п.в. } t \in \Pi,
$$

причем $\left\|Z_{\varepsilon}\right\| \rightarrow 0$ при $\varepsilon \rightarrow+0$.

\section{2. Нелинейный аналог леммы Гронуолла.}

Теорема 2.1. Пусть $B: L_{p} \rightarrow L_{q}, G: L_{q} \rightarrow L_{q}$ - положительные ЛОО, для каждого $\delta>0$ обладающие общей для них вольтерровской $\delta$-цепочкой. Тогда существуют числа $\varepsilon>0, \gamma>0$ такие, что справедливо следующее утверждение. Если $x, \varphi \in L_{q}^{+},\|\varphi\| \leqslant \varepsilon$ и для п.в. $t \in \Pi$ удовлетворяют неравенству

$$
x(t) \leqslant B\left[x^{q / p}\right](t)+G[x](t)+\varphi(t),
$$

mо $x(t) \leqslant S^{-1}[\varphi](t)$ для n.в. $t \in \Pi$, где оператор $S: L_{q} \rightarrow L_{q}$, определяемый формулой

$$
S[x]=x-B\left[x^{q / p}\right]-G[x],
$$

обратим в точке $\varphi$, причем

$$
\left\|S^{-1}[\varphi]\right\| \leqslant \gamma \cdot\|\varphi\| .
$$

\footnotetext{
${ }^{4}$ Как следует из результатов [1], при сделанных предположениях и дополнительных двух условиях, что 1) оператор $\mathscr{G}_{u}$ ограничен при всех $\left.u \in \mathscr{V}, 2\right) \mathscr{T}_{\delta} \subset \mathscr{B}(A)$ при всех $\delta>0$, для того чтобы уравнение (1) имело глобальное решение $y=y_{u}$, достаточно, чтобы число $\varepsilon>0$ было достаточно мало.
} 
ЗАмечаниЕ. Теорема 2.1 может быть существенным образом обобщена. Однако далее при доказательстве основных результатов нам понадобится именно такая формулировка.

3. Доказательство основных результатов. Возможность применения теоремы 2.1 к уравнению (1) основана на следующей лемме, известной как лемма о мажоранте в лебеговом пространстве.

Лемма 3.1. Пусть функиия $f(t, x): \Pi \times \mathbb{R}^{l} \rightarrow \mathbb{R}$ измерима по $t \in \Pi$ и непрерьвна по $x \in \mathbb{R}^{l}$, причем для каждого $y(\cdot) \in L_{q}^{l}(\Pi)$ суперпозииия $f(\cdot, y(\cdot)) \in L_{\sigma}(\Pi)$, где $1 \leqslant q, \sigma<\infty$. Тогда найдутся число $b \geqslant 0$ и функиия $a(\cdot) \in L_{\sigma}^{+}(\Pi)$ такие, что

$$
|f(t, x)| \leqslant a(t)+b \cdot|x|^{q / \sigma} \quad \text { для п.в. } t \in \Pi, \quad x \in \mathbb{R}^{l} .
$$

Для случая $l=1$ лемма 3.1 формулируется и доказывается в [10; лемма 5.17.6, c. 349]. Однако анализ доказательства этой леммы показывает, что одномерность $(l=1)$ используется лишь при ссылке на следующее утверждение (см. там же, лемма 5.17.2).

Лемма 3.2. Пусть П $\subset \mathbb{R}^{n}$ - измеримое по Лебегу ограниченное множество, вектор-функиия $f(t, \xi): \Pi \times \mathbb{R} \rightarrow \mathbb{R}$ - измерима по $t \in \Pi$ и непрерьвна по $\xi \in \mathbb{R}$, a скалярные функиии $a(t), b(t)$ измеримы на $\Pi, a(t) \leqslant b(t)$ для n.в. $t \in \Pi$. Тогда существует измеримая функиия $\theta(t)$, принимающая значения из $[a(t) ; b(t)]$ для п.в. $t \in \Pi$ и такая, что

$$
\max _{\xi \in[a(t) ; b(t)]} f(t, \xi)=f(t, \theta(t)) \quad \text { для n.в. } t \in \Pi .
$$

Однако лемма 3.2 допускает следующее многомерное обобщение.

Лемма 3.3. Пусть $S(\Pi)$ - пространство измеримых почти всюду конечных функиий на $\Pi, l \in \mathbb{N}, a(\cdot), b(\cdot) \in S^{l}(\Pi)$ - измеримые на $\Pi l$-вектор-функции, причем $a(t) \leqslant b(t)$ для n.в. $t \in \Pi$, а функиия $\Phi(t, y): \Pi \times \mathbb{R}^{l} \rightarrow \mathbb{R}$ измерима по $t \in \Pi u$ непрерьвна по $y \in \mathbb{R}^{l}$. Тогда функиия

$$
\varphi(t) \equiv \max _{y \in[a(t) ; b(t)]} \Phi(t, y)
$$

измерима на П, и более того, существует функция $\theta(\cdot)$ из семейства

$$
M[a ; b] \equiv\left\{y \in S^{l}(\Pi): y(t) \in[a(t) ; b(t)]\right\}
$$

такая, что

$$
\Phi(t, \theta(t))=\varphi(t) \quad \text { для n.в. } t \in \Pi .
$$

ЗАмечАниЕ. Для $a, b \in \mathbb{R}^{l}$ неравенство $a \leqslant b$ здесь и далее понимается покомпонентно: $a_{j} \leqslant b_{j}, j=1, \ldots, l$; кроме того, используется обозначение

$$
[a ; b]=\left[a_{1} ; b_{1}\right] \times \cdots \times\left[a_{l} ; b_{l}\right] .
$$

Доказательство леммы 3.3 следует, например, непосредственно из [11; предложение Д1.2, с. 326, и теорема Д1.4, с. 327]. Таким образом, получаем, что справедлива также и лемма 3.1. Отсюда следует 
Лемма 3.4. Пусть функиия $f(t, x, v): \Pi \times \mathbb{R}^{l} \times \mathbb{R}^{s} \rightarrow \mathbb{R}$ измерима по $t \in \Pi u$ непрерывна по $\{x, v\} \in \mathbb{R}^{l+s}$, причем для всех $y(\cdot) \in L_{q}^{l}(\Pi) u u(\cdot) \in L_{r}^{s}(\Pi)$ имеем $f(\cdot, y(\cdot), u(\cdot)) \in L_{\sigma}(\Pi)$, где $1 \leqslant q, r, \sigma<\infty$. Тогда найдутся число $b \geqslant 0$ и функция $a(\cdot) \in L_{\sigma}^{+}(\Pi)$ maкue, чmo

$$
|f(t, x, v)| \leqslant a(t)+b \cdot|x|^{q / \sigma}+b \cdot|v|^{r / \sigma} \quad \text { для n.в. } t \in \Pi, \quad x \in \mathbb{R}^{l}, \quad v \in \mathbb{R}^{s} .
$$

ДокАЗАтельство. Заметим, во-первых, что функция $\psi: \mathbb{R} \rightarrow \mathbb{R}$, определяемая формулой $\psi(z)=\operatorname{sign}(z)|z|^{q / r}$, непрерывна. В таком случае функция

$$
h(t, x, z)=f\left(t, x, \operatorname{sign}\left(z_{1}\right)\left|z_{1}\right|^{q / r}, \ldots, \operatorname{sign}\left(z_{s}\right)\left|z_{s}\right|^{q / r}\right): \Pi \times \mathbb{R}^{l+s} \rightarrow \mathbb{R}
$$

измерима по $t \in \Pi$ и непрерывна по $\{x, z\} \in \mathbb{R}^{l+s}$. Заметим, во-вторых, что если $z(\cdot) \in L_{q}(\Pi)$, то $\operatorname{sign}(z)|z|^{q / r} \in L_{r}(\Pi)$. Таким образом,

$$
h(\cdot, x(\cdot), z(\cdot)) \in L_{\sigma}(\Pi) \quad \text { для всех } \quad\{x, z\} \in L_{q}^{l+s}(\Pi) .
$$

Поэтому согласно лемме 3.1 найдутся $a(\cdot) \in L_{\sigma}^{+}(\Pi)$ и $c \geqslant 0$ такие, что

$$
|h(t, x, z)| \leqslant a(t)+c \cdot|\{x, z\}|^{q / \sigma} \leqslant a(t)+c \cdot|x|^{q / \sigma}+c \sum_{i=1}^{s}\left|z_{i}\right|^{q / \sigma}
$$

для п.в. $t \in \Pi$, для всех $\{x, z\} \in \mathbb{R}^{l+s}$. Остается взять в качестве $z_{i}=\operatorname{sign}\left(v_{i}\right)\left|v_{i}\right|^{r / q}$ и положить $b=s c$. Лемма доказана.

ДокАЗАТЕЛЬСтво тЕоРЕмы 1.1. Следующая оценка очевидна:

$$
|\Delta y| \leqslant B\left[\left|g\left(\cdot, y_{u}, u\right)-g\left(\cdot, y_{v}, u\right)\right|\right]+B\left[\left|\Delta_{u} g\left(y_{v}\right)\right|\right] .
$$

Используя далее теорему Лагранжа о конечных приращениях в интегральной форме, получаем

$$
|\Delta y| \leqslant B\left[\int_{0}^{1}\left|g_{x}^{\prime}\left(\cdot, y_{v}+\theta \Delta y, u\right)\right| d \theta|\Delta y|\right]+B\left[\left|\Delta_{u} g\left(y_{v}\right)\right|\right] .
$$

Применяя лемму 3.4 к функции $f(t, x, z)=\left|g_{x}^{\prime}\left(\cdot, y_{v}+x, z\right)\right|$, получаем, что существуют $a(\cdot) \in L_{\sigma}^{+}(\Pi)$ и $b \geqslant 0$ такие, что

$$
\left|g_{x}^{\prime}\left(\cdot, y_{v}+\theta \Delta y, u\right)\right| \leqslant a(t)+b\left(|\Delta y|^{q / \sigma}+|u|^{r / \sigma}\right) \leqslant a_{0}(t)+b \cdot|\Delta y|^{q / \sigma},
$$

где $a_{0}=a+b|V|^{r / \sigma} \in L_{\sigma}(\Pi)$. Поскольку $(q / \sigma)+1=q / p$, то

$$
|\Delta y| \leqslant b B\left[|\Delta y|^{q / p}\right]+B\left[a_{0}(\cdot)|\Delta y|\right]+B\left[\left|\Delta_{u} g\left(y_{v}\right)\right|\right] .
$$

Остается применить теорему 2.1. Теорема доказана.

ДоКАЗАТЕЛЬСТво тЕОРЕмЫ 1.2. Аналогично доказательству теоремы 1.1 получаем

$$
|\Delta y| \leqslant b B\left[|\Delta y|^{q / p}\right]+B\left[a_{0}(\cdot)|\Delta y|\right]+z_{\varepsilon}
$$

Обозначим

$$
Z(t)=B[z](t), \quad z(t)=\max _{\xi \in \mathbb{R}^{s}:|\xi| \leqslant V(t)}\left|g\left(t, y_{v}(t), \xi\right)-g\left(t, y_{v}(t), v(t)\right)\right| .
$$


Очевидно, что $z_{\varepsilon} \leqslant Z$ и по лемме $3.2 z(\cdot) \in L_{p}^{+}$, а значит, $Z(\cdot) \in L_{q}^{+}$, и согласно [12; теорема I.6.17, с. 68] $z_{\varepsilon}$ - измеримая функция. Тогда в силу идеальности пространства $L_{q}, z_{\varepsilon} \in L_{q}^{+}$. По условию и согласно теореме Лебега о предельном переходе под знаком интеграла получаем, что $\left\|z_{\varepsilon}\right\| \rightarrow 0$ при $\varepsilon \rightarrow+0$. Остается воспользоваться теоремой 2.1 и положить $Z_{\varepsilon}=S^{-1}\left[z_{\varepsilon}\right]$. Теорема доказана.

4. Доказательство нелинейного аналога леммы Гронуолла. Сначала докажем два вспомогательных утверждения, которые касаются свойств нелинейного уравнения вида

$$
y=B[b(y)]+G[g(y)]+\varphi, \quad y \in L_{q}(\Pi),
$$

где предполагается, что $B: L_{p} \rightarrow L_{q}, G: L_{r} \rightarrow L_{q}$ - положительные линейные ограниченные операторы, для любого $\delta>0$ обладающие общей для них вольтерровской $\delta$-цепочкой $\mathscr{T},+\infty>q \geqslant \max \{p, r\}, p, r \in[1,+\infty) ; b(y), g(y)$ - дифференцируемые функции такие, что

1) $b(x) \in L_{p}^{+}$для всех $x \in L_{q}^{+}, b(0)=0$;

2) $b^{\prime}(x) \in L_{\sigma}^{+}$, где $\sigma=(p q) /(q-p)$, если $q>p$, или $\sigma=+\infty$, если $q=p$;

3) существует неубывающая функция $\mathscr{N}_{b}: \mathbb{R}_{+} \rightarrow \mathbb{R}_{+}$такая, что

$$
\left\|b^{\prime}(x)\right\|_{L_{\sigma}} \leqslant \mathscr{N}_{b}(\|x\|) \quad \text { для всех } \quad x \in L_{q}^{+} ;
$$

4) $g(x) \in L_{r}^{+}$для всех $x \in L_{q}^{+} ; g(0)=0$;

5) $g^{\prime}(x) \in L_{\nu}^{+}$, где $\nu=(r q) /(q-r)$, если $q>r$, или $\nu=+\infty$, если $q=r$;

6 ) существует неубывающая функция $\mathscr{N}_{g}: \mathbb{R}_{+} \rightarrow \mathbb{R}_{+}$такая, что

$$
\left\|g^{\prime}(x)\right\|_{L_{\nu}} \leqslant \mathscr{N}_{g}(\|x\|) \quad \text { для всех } \quad x \in L_{q}^{+} .
$$

В частности, для функции $b(x)=x^{q / p}$ при условии, что $q>p$, можем взять

$$
\mathscr{N}_{b}(t)=\frac{q}{p} t^{(q-p) / p}, \quad t \geqslant 0 .
$$

Если же $q=p$, то $\mathscr{N}_{b}(x) \equiv 1$.

Определим оператор $F: L_{q}^{+} \rightarrow L_{q}^{+}$формулой

$$
F[y]=B[b(y)]+G[g(y)]+\varphi .
$$

Пусть $H_{1}, H_{2}$ - два соседних множества вольтерровской $\delta$-цепочки $\mathscr{T}, H_{1} \subset H_{2}$, $h=H_{2} \backslash H_{1}$. Для произвольных $x, z, z+\Delta z \in L_{q}^{+}$рассмотрим норму

$$
\left\|P_{h}\left(F\left[P_{H_{1}} x+P_{h} z\right]-F\left[P_{H_{1}} x+P_{h}[z+\Delta z]\right]\right)\right\| .
$$

Пользуясь теоремой Лагранжа о конечных приращениях в интегральной форме, а также неравенством Гёльдера, нетрудно показать, что для этой нормы справедлива оценка

$$
\begin{aligned}
\|\ldots\| \leqslant\left(\left\|P_{h} B P_{h}\right\| \cdot \sup _{\theta \in[0 ; 1]}\left\|b^{\prime}\left(P_{H_{1}} x+\theta P_{h} \Delta z\right)\right\|_{L_{\sigma}}\right. \\
\left.\quad+\left\|P_{h} G P_{h}\right\| \cdot \sup _{\theta \in[0 ; 1]}\left\|g^{\prime}\left(P_{H_{1}} x+\theta P_{h} \Delta z\right)\right\|_{L_{\kappa}}\right) \cdot\left\|P_{h} \Delta z\right\|,
\end{aligned}
$$


откуда по определению вольтерровской $\delta$-цепочки и по условиям 3$)$ и 6 ) на функции $b(\cdot)$ и $g(\cdot)$ получаем

$$
\|\cdots\| \leqslant \delta \cdot \sup _{\theta \in[0 ; 1]} \mathscr{N}\left(\left\|P_{H_{1}} x+\theta P_{h} \Delta z\right\|\right)\left\|P_{h} \Delta z\right\|,
$$

где $\mathscr{N}=2\left(\mathscr{N}_{b}+\mathscr{N}_{g}\right)$. Оценку $(*)$ далее мы будем неоднократно использовать. Следующие две оценки с использованием условия $b(0)=g(0)=0$, см. 1), 4), получаются точно таким же методом:

$$
\|b(x)\|_{L_{p}} \leqslant \mathscr{N}_{b}(\|x\|) \cdot\|x\|, \quad\|g(x)\|_{L_{r}} \leqslant \mathscr{N}_{g}(\|x\|) \cdot\|x\| \quad \text { для всех } \quad x \in L_{q}^{+} . \quad \text { (**) }
$$

Доказательство следующих далее лемм 4.1 и 4.2 основано на идеях [1].

ЛЕмма 4.1. Ни при каком $\varphi(\cdot) \in L_{q}$ уравнение (2) не может иметь более одного решения в $L_{q}^{+}(\Pi)$.

ДоКАЗАТЕЛЬСтво. Предположим, от противного, что существуют два решения $y_{1}, y_{2} \in L_{q}^{+}$. Положим $\Delta y=y_{2}-y_{1}, K=\max _{i=1,2}\left\|y_{i}\right\|$. Выберем теперь число $\delta>0$ из условия

$$
\delta \leqslant \frac{1}{2 \mathscr{N}(2 K)} .
$$

Согласно условию операторы $B$ и $G$ имеют общую для них вольтерровскую $\delta$-цепочку $\left\{H_{0}, H_{1}, \ldots, H_{k}\right\}$. Обозначим для краткости $P_{i}=P_{H_{i}}, P_{(i, j)}=P_{h}$, где $h=H_{j} \backslash H_{i}$. Заметим, что при всех $i=1, \ldots, k P_{i} y_{1}$ и $P_{i} y_{2}$ являются решениями уравнения

$$
P_{i} y=P_{i} F P_{i}[y],
$$

представляющего собой локальный аналог уравнения (2). Собственно говоря, их можно понимать как $H_{i}$-локальные решения этого уравнения. Далее индукцией по $i$ мы покажем, что $P_{i} \Delta y=0, i=1, \ldots, k$. Это и будет означать, что имеет место доказываемое утверждение.

1. Пользуясь оценкой $(*)$, немедленно получаем

$$
\left\|P_{1} \Delta y\right\| \leqslant \delta \sup _{\theta \in[0 ; 1]} \mathscr{N}\left(\left\|y_{\theta}\right\|\right) \cdot\left\|P_{1} \Delta y\right\|
$$

где

$$
\left\|y_{\theta}\right\|=\left\|(1-\theta) y_{1}+\theta y_{2}\right\| \leqslant(1-\theta)\left\|y_{1}\right\|+\theta\left\|y_{2}\right\| \leqslant K .
$$

Тогда в силу выбора $\delta$ находим оценку

$$
\left\|P_{1} \Delta y\right\| \leqslant \frac{1}{2}\left\|P_{1} \Delta y\right\|
$$

которая возможна только в одном случае: $P_{1} \Delta y=0$.

2. Действуя по индукции, предположим, что $P_{m} \Delta y=0$. Пользуясь снова оценкой $(*)$, совершенно аналогично п. 1 этого доказательства получаем

$$
\left\|P_{(m, m+1)} \Delta y\right\| \leqslant \delta \mathscr{N}(2 K) \cdot\left\|P_{(m, m+1)} \Delta y\right\| \leqslant \frac{1}{2}\left\|P_{(m, m+1)} \Delta y\right\|,
$$

а это означает, что

$$
P_{m+1} \Delta y=P_{(m, m+1)} \Delta y=0 .
$$

Лемма доказана. 
Лемма 4.2. Существуют числа $\varepsilon>0 u \gamma>0$ такие, что для всякого $\varphi \in U_{\varepsilon}(0)$, əəe

$$
U_{\varepsilon}(0) \equiv\left\{\varphi \in L_{q}^{+}:\|\varphi\| \leqslant \varepsilon\right\},
$$

уравнение (2) имеет единственное решение $y \in L_{q}^{+}$, причем $\|y\| \leqslant \gamma\|\varphi\|$. Более того, если для некоторого $\varphi=\psi \in L_{q}$ уравнение (2) имеет решение $y=x \in L_{q}^{+}$, то для каждого $\varphi \in U_{\varepsilon}(0), \varphi \geqslant \psi($ или $\varphi \leqslant \psi)$, отвечающее ему решение $y \in L_{q}^{+}$ удовлетворяет неравенству $y \geqslant x$ (соответственно $y \leqslant x)$.

ДокАзАТЕЛЬСтво. Выберем число $\delta>0$ следующим образом:

$$
\delta=\frac{1}{2 \mathscr{N}(4)} .
$$

Согласно условию операторы $B$ и $G$ имеют общую для них вольтерровскую $\delta$-цепочку $\left\{H_{0}, H_{1}, \ldots, H_{k}\right\}$. Соответственно, определим числа

$$
\gamma=C_{k}, \quad \varepsilon=\frac{1}{C_{k} \mathscr{N}\left(C_{k}\right)(\|B\|+\|G\|)+1},
$$

где $C_{j}$ - числовая последовательность, определяемая рекуррентной формулой

$$
C_{j+1}=C_{j}+2\left\{C_{j} \mathscr{N}\left(C_{j}\right)(\|B\|+\|G\|)+1\right\}, \quad C_{0}=0, \quad C_{1}=2 .
$$

Итак, предположим, что уравнение (2) имеет решение $y=x \in L_{q}^{+}$при некотором $\varphi=\psi \in L_{q}$. Заметим, что такие $x$ и $\psi$ заведомо существуют, в частности, $x=0$ и $\psi=0$ (более того, их множество бесконечно, поскольку по всякому $x \in L_{q}^{+}$можно найти соответствующее $\psi$ ). Зафиксируем любое $\varphi \in U_{\varepsilon}(0)$ такое, что $\varphi \geqslant \psi$ (при $\psi=0$ такая $\varphi$ заведомо существует). В случае неравенства "\$” все дальнейшие рассуждения аналогичны. Рассматривая уравнение $\left(E_{i}\right)$, докажем наше утверждение по индукции для $i=1, \ldots, k$. При этом будем использовать обозначения $P_{i}, P_{(i, j)}$, введенные ранее при доказательстве предыдущей леммы, и, кроме того, будем полагать $P L_{q}^{+}=\left\{y \in L_{q}^{+}: y=P y\right\}$.

1. Определим множество

$$
Y=\left\{y \in P_{1} L_{q}^{+}: y \geqslant P_{1} x,\|y\| \leqslant 2\right\}
$$

а) Возьмем любое $y \in Y$ и покажем, что

$$
P_{1} F[y]=P_{1} F P_{1}[y] \in Y
$$

В силу положительности операторов $B$ и $G$, а также учитывая, что функции $b(\cdot)$ и $g(\cdot)$ согласно накладываемым на них условиям не убывают, можем оценить

$$
P_{1} F[y] \geqslant P_{1} B P_{1}[b(x)]+P_{1} G P_{1}[g(x)]+P_{1} \psi=P_{1} x .
$$

Оценим норму

$$
\left\|P_{1} F[y]\right\| \leqslant\left\|P_{1} B P_{1}\right\| \cdot\|b(y)\|_{L_{p}}+\left\|P_{1} G P_{1}\right\| \cdot\|g(y)\|_{L_{r}}+\|\varphi\| .
$$


Пользуясь далее неравенствами $(* *)$ и учитывая, что $\|y\| \leqslant 2,\|\varphi\| \leqslant \varepsilon \leqslant 1$, и в силу выбора $\delta$ получаем

$$
\left\|P_{1} F[y]\right\| \leqslant 2 \delta\left(\mathscr{N}_{b}(2)+\mathscr{N}_{g}(2)\right)+1 \leqslant 1+1=2 .
$$

Таким образом, $F[y] \in Y$.

б) Проверим, что $F$ - сжимающий на $Y$. Для любых $y_{1}, y_{2} \in Y$ аналогично доказательству леммы 4.1 (при $K=2$ ) можем оценить

$$
\left\|P_{1} F \Delta y\right\| \leqslant \delta \mathscr{N}(2) \cdot\left\|P_{1} \Delta y\right\| \equiv \alpha \cdot\left\|P_{1} \Delta y\right\|,
$$

и в силу выбора $\delta$ имеем: $\alpha<1$.

Поскольку из сходимости по норме $L_{q}$ следует сходимость по мере, а из сходимости последовательности по мере по теореме Ф. Рисса следует существование подпоследовательности, сходящейся почти всюду, то нетрудно понять, что множество $Y$ замкнуто. Пользуясь далее принципом неподвижной точки Каччопполи-Банаха (см., например, [12]), получаем, что решение уравнения $\left(E_{1}\right)$ существует и единственно на множестве $Y$, обозначим его $y_{1}=P_{1} y_{1}=P_{1} F y_{1}$. Аналогично пункту а) получаем для него оценку

$$
\left\|y_{1}\right\| \leqslant \delta \cdot\|b(y)\|_{L_{p}}+\delta \cdot\|g(y)\|_{L_{r}}+\|\varphi\| \leqslant \delta\left\|y_{1}\right\|\left(\mathscr{N}_{b}(2)+\mathscr{N}_{g}(2)\right)+\|\varphi\|
$$

и в силу выбора $\delta$

$$
\left\|y_{1}\right\| \leqslant \frac{1}{2}\left\|y_{1}\right\|+\|\varphi\|
$$

откуда

$$
\left\|y_{1}\right\|=\left\|P_{1} y_{1}\right\| \leqslant 2\|\varphi\|=C_{1}\|\varphi\| .
$$

2. Действуя по индукции, предположим, что уравнение $\left(E_{i}\right)$ при $i=m$ имеет решение $y_{m}=P_{m} y_{m}$, причем $\left\|y_{m}\right\| \leqslant C_{m}\|\varphi\|, y_{m} \geqslant P_{m} x$. Докажем, что такое же утверждение выполняется и при $i=m+1$. Заметим, что нам достаточно рассмотреть уравнение

$$
y=P_{(m, m+1)} B P_{(m, m+1)}[b(y)]+P_{(m, m+1)} G P_{(m, m+1)}[g(y)]+\varphi_{m+1} \quad\left(E_{m+1}^{\prime}\right)
$$

относительно $y=P_{(m, m+1)} y \in P_{(m, m+1)} L_{q}^{+}$, где принято обозначение

$$
\varphi_{m+1}=P_{(m, m+1)} B P_{m}[b(y)]+P_{(m, m+1)} G P_{m}[g(y)]+P_{(m, m+1)} \varphi .
$$

Действительно, если оно имеет решение $y=P_{(m, m+1)} y$, то $y_{m+1}=y_{m}+y$ является решением уравнения $\left(E_{i}\right)$ при $i=m+1$. Кроме того, заметим, что $P_{(m, m+1)} x$ является решением аналогичного уравнения при замене $\varphi_{m+1}$ на

$$
\psi_{m+1}=P_{(m, m+1)} B P_{m}[b(x)]+P_{(m, m+1)} G P_{m}[g(x)]+P_{(m, m+1)} \psi \leqslant \varphi_{m+1}
$$

согласно предположению индукции. При этом

$$
\left\|\varphi_{m+1}\right\| \leqslant\left\{\mid B\left\|\cdot C_{m} \mathscr{N}_{b}\left(C_{m}\|\varphi\|\right)+\right\| G \| \cdot C_{m} \mathscr{N}_{g}\left(C_{m}\|\varphi\|\right)+1\right\}\|\varphi\|,
$$

а поскольку $\|\varphi\| \leqslant \varepsilon \leqslant 1$, то

$$
\left\|\varphi_{m+1}\right\| \leqslant\left\{\|B\| \cdot C_{m} \mathscr{N}_{b}\left(C_{m}\right)+\|G\| \cdot C_{m} \mathscr{N}_{g}\left(C_{m}\right)+1\right\}\|\varphi\| \leqslant\{\ldots\} \varepsilon \leqslant 1
$$

в силу выбора $\varepsilon$ и монотонности последовательности $C_{j}$. 
Поскольку в пункте 1 этого доказательства мы не использовали относительно $x$, $\psi$ и $\varphi$ никакой иной информации, то аналогично пункту 1 показываем, что уравнение $\left(E_{m+1}^{\prime}\right)$ имеет решение $P_{(m, m+1)} y \in P_{(m, m+1)} L_{q}^{+}$, причем $P_{(m, m+1)} y \geqslant P_{(m, m+1)} x$;

$$
\left\|P_{(m, m+1)} y\right\| \leqslant 2\left\|\varphi_{m+1}\right\| \leqslant 2\left\{\|B\| \cdot C_{m} \mathscr{N}_{b}\left(C_{m}\right)+\|G\| \cdot C_{m} \mathscr{N}_{g}\left(C_{m}\right)+1\right\}\|\varphi\|,
$$

следовательно, уравнение $\left(E_{m+1}\right)$ имеет решение

$$
\begin{aligned}
y_{m+1} & =P_{m} y_{m}+P_{(m, m+1)} y \geqslant P_{m} x+P_{(m, m+1)} x=P_{m+1} x, \\
\left\|y_{m+1}\right\| & \leqslant C_{m}\|\varphi\|+2\left\{C_{m} \mathscr{N}\left(C_{m}\right)[\|B\|+\|G\|]+1\right\}\|\varphi\|=C_{m+1}\|\varphi\| .
\end{aligned}
$$

Таким образом, имеет место доказываемое утверждение при $i=m+1$. В силу леммы 4.1 решение $y_{k}$, которое мы получаем на последнем шаге индукции, будет единственным решением уравнения (2) во всем $L_{q}^{+}$. Лемма доказана.

ДоКАЗАТЕЛЬСТво тЕОРЕмЫ 2.1. Возьмем $\varepsilon>0$ и $\gamma>0$ из формулировки леммы 4.2. Положим

$$
r=q, \quad b(x)=x^{q / p}, \quad g(x)=x, \quad \psi=x-B\left[x^{q / p}\right]-G[x] \in L_{q} .
$$

Тогда $y=x$ является решением уравнения (2) при $\varphi=\psi$. По условию $\psi \leqslant \varphi$, $\varphi \in U_{\varepsilon}(0)$. Тогда по лемме 4.2 уравнение $(2)$, т.е. $S[y]=\varphi$, имеет решение $y \in L_{q}^{+}$, причем $x \leqslant y=S^{-1}[\varphi],\left\|S^{-1}[\varphi]\right\|=\|y\| \leqslant \gamma\|\varphi\|$. Теорема доказана.

5. Пример. Чтобы пояснить применение доказанных результатов к исследованию управляемых начально-краевых задач, рассмотрим следующий иллюстративный пример. Пусть $n=2, T_{0}>0 ; T^{\prime}$ - некоторая фиксированная точка в пространстве $\mathbb{R}^{3}$ переменных $t=\left\{t_{0}, \bar{t}\right\}$ такая, что $T_{0}^{\prime} \geqslant T_{0} ; K\left(T^{\prime}\right)$ - конус (характеристический конус прошлого для следующего далее волнового уравнения) с вершиной в точке $T^{\prime}$ и основанием

$$
Q=\left\{\bar{t} \in \mathbb{R}^{3}:\left|\bar{t}-\overline{T^{\prime}}\right|<T_{0}^{\prime}\right\}
$$

на плоскости $t_{0}=0$ ( $t_{0}-$ время, $\bar{t}-$ пространственные переменные); П - часть конуса $K\left(T^{\prime}\right)$, попадающая в полосу $0 \leqslant t_{0} \leqslant T_{0}$ (предполагаем, что $\Pi=\bar{\Pi}$ ); $\partial Q-$ граница $Q, \partial \Pi$ - граница $\Pi, \Gamma=\partial \Pi \backslash\left\{t \in \mathbb{R}^{3}: t_{0}=0\right\}$. Поставим для $w_{1} \in H_{0}^{1}(Q)$, $w_{2} \in L_{2}(Q)$ задачу Коши на множестве П:

$$
\begin{gathered}
\mathscr{L}[x](t) \equiv \frac{\partial^{2} x}{\partial t_{0}^{2}}-\sum_{j=1}^{n} \frac{\partial^{2} x}{\partial t_{j}^{2}}=x^{3}(t)+x(t) u(t), \quad u \in U \subset L_{2}(\Pi), \\
x(0, \bar{t})=w_{1}(\bar{t}), \quad x_{t_{0}}^{\prime}(0, \bar{t})=w_{2}(\bar{t}), \quad \bar{t} \in Q,\left.\quad x\right|_{\Gamma}=0 .
\end{gathered}
$$

Рассмотрим задачу (4) для вспомогательного уравнения

$$
\mathscr{L}[x](t)=z(t), \quad t \in \Pi, \quad z(\cdot) \in L_{3 / 2}(\Pi) .
$$

Решение задачи (5), (4) понимаем как обобщенное в слабом смысле:

$$
\begin{gathered}
\int_{\Pi} x(t) \mathscr{L}[\varphi](t) d t=\int_{\Pi} z(t) \varphi(t) d t+\int_{Q}\left[\varphi(0, \bar{t}) w_{2}(\bar{t})-\frac{\partial \varphi}{\partial t_{0}}(0, \bar{t}) w_{1}(\bar{t})\right] d \bar{t}, \\
\left.\varphi\right|_{\Gamma}=0, \quad \varphi_{t_{0}}^{\prime}\left(T_{0}, \bar{t}\right)=0 \quad \text { для всех } \varphi \in \mathbb{C}^{2}(\Pi) .
\end{gathered}
$$


Соответственно, решение задачи (3), (4) понимаем как решение задачи (5), (4) при $z=x^{3}+x u$.

Обозначим $A[z]$ - решение задачи (5), (4) при $w_{1} \equiv w_{2} \equiv 0$, а $\Theta\left[w_{1}, w_{2}\right]$ - решение той же задачи при $z \equiv 0$. Положим $Q_{\tau}$ - сечение множества П плоскостью $t_{0}=\tau$,

$$
\mathscr{H}=\mathscr{H}(\Pi)=L_{\infty}\left(\tau \in\left[0, T_{0}\right] ; H^{1-n / 6}\left(Q_{\tau}\right)\right) .
$$

Из [13; теорема 2.2, лемма 3.1 , теорема 3.1$]^{5}$ следует, что определенный выше оператор $A$ можно рассматривать как ЛОО $L_{3 / 2}(\Pi) \rightarrow \mathscr{H}(\Pi)$, а $\theta=\Theta\left[w_{1}, w_{2}\right] \in \mathscr{H}(\Pi)$.

Известно (см. [14; замечание 1 к теореме 4.6.1, теорема 4.6.2]), что при $n=1,2$ пространство $\mathscr{H}(\Pi)$ ограниченно вложено в $L_{6}(\Pi)$. Поэтому $A: L_{3 / 2}(\Pi) \rightarrow L_{6}(\Pi)-$ ЛОО, $\theta \in L_{6}(\Pi)$, и задачу (3), (4) можно записать как уравнение $(1)$ при $g(t, x, u)=$ $x^{3}+x u, p=3 / 2, q=6, s=2$. Далее мы покажем, что все условия, которые мы накладывали на это уравнение при доказательстве теорем 1.1, 1.2 выполнены. Результат применения этих теорем очевиден.

Понятно, что условия K) выполнены. Таким образом, нам достаточно проверить условия А). Покажем, во-первых, что оператор $A$ является положительным, и таким образом, сам для себя является мажорантой: $B=A$. В связи с этим заметим, что понятие обобщенного в слабом смысле решения является частным случаем понятия решения в терминах обобщенных функций (понимаемых здесь как интегральные функционалы). Поэтому, пользуясь теоремой [15; п. 3.3.3], заключаем, что решение $A[z]$ как обобщенное решение (будем обозначать его $\{A[z]\}$ ) представляется в виде свертки $\{A[z]\}=\mathscr{E}_{2} * z$ (волнового потенциала), где $\mathscr{E}_{2}-$ фундаментальное решение волнового оператора. Пусть далее $z(\cdot) \in L_{3 / 2}(\Pi), z(\cdot) \geqslant 0,-$ фиксировано (в соответствии с общей методикой приведения задачи Коши к задаче в терминах обобщенных функций мы продолжаем $A[z]$ и $z$ на все пространство $\mathbb{R}^{3}$ нулем). Как уже сказано, $A[z] \in L_{6}(\Pi)$, т.е. во всяком случае является суммируемой функцией. Как известно, $\mathscr{E}_{2}$ - тоже суммируемая функция, причем $\mathscr{E}_{2}(\cdot) \geqslant 0$. Таким образом, можем записать:

$$
\begin{aligned}
(\{A[z]\}, \varphi) & =\int_{\Pi} A[z](t) \varphi(t) d t=\left(\mathscr{E}_{2} * z, \varphi\right) \\
& =\left(\mathscr{E}_{2}(t) \cdot z(\tau), \varphi(t+\tau)\right)=\int_{\Pi} z(\tau)\left(\int_{\mathbb{R}^{3}} \mathscr{E}_{2}(t) \varphi(t+\tau) d t\right) d \tau .
\end{aligned}
$$

Отсюда видно, что если $z(\tau) \geqslant 0$, то для всех основных функций $\varphi(\cdot) \geqslant 0$ имеем

$$
\int_{\Pi} A[z](t) \varphi(t) d t \geqslant 0 .
$$

Пользуясь леммой 5.1 (см. ниже), заключаем, что $A[z](t) \geqslant 0$, т.е. оператор $A$ положительный.

Лемма 5.1. Пусть $\Pi \subset \mathbb{R}^{n}-$ компакт, $x(\cdot) \in L(\Pi) u \int_{\Pi} x(t) \varphi(t) d t \geqslant 0 \quad \partial \Omega я$ любых $\varphi(\cdot) \in D(\Pi), \varphi(\cdot) \geqslant 0$, где $D(\Pi)$ - множество всех основных функиий с носителем в П. Тогда $x(t) \geqslant 0$ для n.в. $t \in \Pi$.

\footnotetext{
${ }^{5}$ На самом деле, там рассматривается смешанная задача на цилиндре с нулевыми данными на боковой поверхности, но понятно, что отсюда получается аналогичный результат и для задачи Коши на характеристическом конусе прошлого.
} 
ДоказАТЕЛЬство. Предположим, нашлось множество $\Pi_{0} \subset$ П такое, что

$$
\operatorname{mes}\left(\Pi_{0}\right)>0, \quad x(t)<0, \quad t \in \Pi_{0} .
$$

Тогда $\int_{\Pi_{0}} x(t) d t=-\gamma<0$. Поскольку множество П измеримо, то, как известно, для каждого $\varepsilon>0$ найдется открытое множество $\Pi_{\varepsilon} \supset \Pi_{0}$ такое, что $\operatorname{mes}\left(\Pi_{\varepsilon} \backslash \Pi_{0}\right)<\varepsilon$. Но в силу абсолютной непрерывности интеграла Лебега

$$
\int_{\Pi_{\varepsilon} \backslash \Pi_{0}}|x(t)| d t \rightarrow 0 \quad \text { при } \quad \varepsilon \rightarrow+0 .
$$

Поэтому существует открытое множество $\Pi^{\prime} \supset \Pi_{0}, \Pi^{\prime} \subset \Pi$, такое, что

$$
\gamma_{1}=\int_{\Pi^{\prime}} x(t) d t \leqslant-\frac{\gamma}{2}<0
$$

Открытое ограниченное множество можно, как известно, представить в виде счетного объединения замкнутых квадратов, не имеющих попарно общих внутренних точек. В силу полной аддитивности интеграла Лебега интеграл от функции $x(t)$ по крайней мере по одному из этих квадратов должен быть отрицательным. Поэтому можем считать, что найдется открытый квадрат $Q_{0} \subset \Pi^{\prime}$ :

$$
\gamma_{2}=\int_{Q_{0}} x(t) d t<0
$$

Рассмотрим бесконечную систему вложенных друг в друга открытых квадратов

$$
Q_{1} \subset Q_{2} \subset \cdots \subset Q_{0}, \quad \operatorname{mes}\left(Q_{0} \backslash Q_{m}\right) \rightarrow 0 \quad \text { при } \quad m \rightarrow \infty .
$$

Для каждого из них существует (см. [15; п. 2.1.2, с. 70]) основная функция

$$
\varphi_{m}(\cdot) \in D\left(Q_{0}\right), \quad \varphi_{m}(t) \in[0,1], \quad \varphi_{m}(t) \equiv 1, \quad t \in Q_{m} .
$$

Поскольку $Q_{0} \subset$ П, согласно условию леммы имеем

$$
\sigma_{m}=\int_{Q_{0}} x(t) \varphi_{m}(t) d t=\int_{\Pi} x(t) \varphi_{m}(t) d t \geqslant 0, \quad m \in \mathbb{N} .
$$

При этом

$$
\sigma_{m}=\int_{Q_{0}} x(t) \varphi_{m}(t) d t \rightarrow \int_{Q_{0}} x(t) d t=-\gamma_{2}<0 \quad \text { при } \quad m \rightarrow \infty .
$$

Действительно, при $m \rightarrow \infty$

$$
\left|\int_{Q_{0}} x(t) \varphi_{m}(t) d t-\int_{Q_{0}} x(t) d t\right|=\left|\int_{Q_{0} \backslash Q_{m}} x(t) \varphi_{m}(t) d t\right| \leqslant \int_{Q_{0} \backslash Q_{m}}|x(t)| d t \rightarrow 0 .
$$

Таким образом, получаем противоречие. Лемма доказана.

Покажем, наконец, что ЛОО $A$ обладает для любого $\delta>0$ вольтерровской $\delta$-цепочкой. В соответствии с общеизвестными свойствами решений волнового уравнения множества вида $\Pi[\tau]=\Pi \cap\{[0, \tau) \times Q\}$ являются вольтерровскими множествами оператора $A$, так как сужение $A[z]$ на $\Pi[\tau]$ зависит лишь от значений $z$ на $\Pi[\tau]$. 
Зафиксируем любое $\delta>0$ и, пользуясь установленными свойствами оператора $A$, найдем систему множеств $H_{i}=\Pi\left[\tau_{i}\right], i=0, \ldots, k$, так, чтобы

$$
\left\|P_{H_{i} \backslash H_{i-1}} A\right\|_{L\left(L_{3 / 2}, L_{6}\right)}<\delta, \quad i=1, \ldots, k .
$$

Известно [14; замечание 1 к теореме 4.6.1, теорема 4.6.2], что при $n=1,2$ пространство $H^{1-n / 6}(Q)$ ограниченно вложено в $L_{6}(Q)$. Поэтому для любого $z \in L_{3 / 2}$ такого, что $\|z\| \leqslant 1$, имеем

$$
\begin{aligned}
\left\|P_{H_{i} \backslash H_{i-1}} A z\right\|_{L_{6}} & \left.=\left(\int_{\tau_{i-1}}^{\tau_{i}} d t_{0} \| A z\right)\left\{t_{0}, \cdot\right\} \|_{L_{6}\left(Q_{t_{0}}\right)}^{6}\right)^{1 / 6} \\
& \leqslant C\left(\int_{\tau_{i-1}}^{\tau_{i}} d t_{0}\left\|(A z)\left\{t_{0}, \cdot\right\}\right\|_{H^{1-n / 6}\left(Q_{t_{0}}\right)}^{6}\right)^{1 / 6} \\
& \leqslant C\|A[z]\|_{L_{\infty}\left(0, T ; H^{1-n / 6}\left(Q_{t_{0}}\right)\right)} \cdot\left|\tau_{i}-\tau_{i-1}\right|^{1 / 6} \\
& \leqslant C\|A\|_{L\left(L_{3 / 2}, L_{\infty}\left(0, T ; H^{1-n / 6}\left(Q_{t_{0}}\right)\right)\right)} \cdot\left|\tau_{i}-\tau_{i-1}\right|^{1 / 6}<\delta
\end{aligned}
$$

т.е. все сводится к тому, чтобы $\left|\tau_{i}-\tau_{i-1}\right|$ было достаточно мало, $i=1, \ldots, k$.

\section{СПИСОК ЦИТИРОВАННОЙ ЛИТЕРАТУРЫ}

[1] В.И. Сумин, А.В. Чернов, "О достаточных условиях устойчивости существования глобальных решений вольтерровых операторных уравнений”, Вестн. Нижегородск. гос. ун-та им. Н.И. Лобачевского. Сер. матем. модел. и оптим. упр., 26:1 (2003), $39-49$.

[2] Н. В. Азбелев, "О границах применимости теоремы Чаплыгина о дифференциальных неравенствах", Матем. сб., 39:2 (1956), 161-178.

[3] Н. В. Азбелев, З.Б. Цалюк, "Об интегральных неравенствах. I", Матем. сб., 56:3 (1962), 325-342.

[4] Е. С. Жуковский, "Неравенства Вольтерра в функциональных пространствах", Матем. сб., 195:9 (2004), 3-18.

[5] Н. С. Курпель, Б. А. Шувар, Двусторонние операторные неравенства и их применение, Наукова думка, Киев, 1980.

[6] В.И. Сумин, "Функционально-операторные вольтерровы уравнения в теории оптимального управления распределенными системами", Докл. АН CCCP, 305:5 (1989), 1056-1059.

[7] В.И. Сумин, "О функциональных вольтерровых уравнениях", Изв. вузов. Матем., 1995, № 9, 67-77.

[8] В.И. Сумин, "Управляемые функциональные вольтерровы уравнения в лебеговых пространствах", Вестн. Нижегородск. гос. ун-та им. Н.И. Лобачевского. Сер. матем. модел. и оптим. упр., 19:2 (1998), 138-151.

[9] В. И. Сумин, А. В. Чернов, "Операторы в пространствах измеримых функций: вольтерровость и квазинильпотентность", Дифферени. уравнения, 34:10 (1998), 1402-1411.

[10] М. А. Красносельский, П. П. Забрейко, Е. И. Пустыльник, П. Е. Соболевский, Интегральные операторы в пространствах суммируемых функиий, Наука, М., 1966.

[11] Б.Ш. Мордухович, Методы аппроксимаций в задачах оптимизации и управления, Наука, М., 1988.

[12] Л.В.Канторович, Г. П. Акилов, Функииональный анализ, Наука, М., 1984.

[13] Ж.-Л. Лионс, Управление сингулярными распределенными системами, Наука, М., 1987. 
[14] Х. Трибель, Теории интерполяиии, функииональные пространства, дифференииальные операторы, Мир, М., 1980.

[15] В. С. Владимиров, В. В. Жаринов, Уравнения математической физики, Физматлит, M., 2000.

А. В. Чернов

Институт радиоэлектроники и информационных технологий, Нижегородский государственный технический университет

E-mail: chavnn@mail.ru
Поступило

18.06 .2007

Исправленный вариант

10.01.2010 